\title{
Application of narrow band imaging in early screening of colorectal cancer
}

\author{
Qianyi LIU ${ }^{1 *}$ (D), Weishan RUAN ${ }^{1}$, Zhishang LIU' ${ }^{1}$, Jiefeng $\mathrm{LI}^{1}$, Jiayan $\mathrm{LI}^{1}$
}

\begin{abstract}
To explore the clinical application value of narrow band imaging (NBI) in the early screening of colorectal cancer. 200 patients were selected as the research objects, and were divided into test group and control group by the randomization method, within 100 cases in each group. The patients in the test group were examined by NBI, while the patients in the control group were examined by ordinary endoscopy, and then the lesion detection rate, image definition and pathological examination results of the two groups were compared. After the patients in the test group received NBI examination, the lesion detection rate, image definition and lesion detection coincidence rate were significantly higher than those of the control group, and the comparative difference between the two groups was statistically significant $(P<0.05)$. NBI examination of colorectal mucosa can clearly show the pit pattern and the morphological structure of capillaries of the early colorectal cancer and its precancerous lesions, thus effectively improving the diagnosis rate of early colorectal cancer and its precancerous lesions, playing a good clinical application value in the early screening of colorectal cancer, and guiding the later treatment, so it is suitable for clinical promotion.
\end{abstract}

Keywords: narrow band imaging; colorectal cancer; early screening.

Practical Application: As an emerging technology, narrowband imaging technology has been preliminarily applied to the examination of gastrointestinal and head diseases. Major hospitals will give priority to the use of this technology when screening early related diseases, so as to detect related diseases earlier, find and treat early, and improve the cure rate.

\section{Introduction}

Colorectal cancer is a clinically common malignant tumor disease, including colon cancer and rectal cancer, and due to its poor prognosis, it has higher morbidity and mortality (Xu et al., 2018). In the early stage of colorectal cancer, there is generally no obvious clinical symptom, so the patients are not easy to recognize their disease, and most patients are already in the middle and late stages when they are diagnosed. Some patients have even experienced metastasis and infiltration, which have caused changes in the involved organs, thus resulting in disease aggravation and low cure rate (Yan et al., 2016). However, if the disease can be diagnosed and treated timely, the cure rate can still reach up to $90 \%$ or above (Tu, 2019). Therefore, at present, the most clinically important problem that most urgently needs to be resolved is to actively find ways to improve the early diagnosis rate of colorectal cancer, thus reducing the mortality of patients with colorectal cancer, improving the prognosis of patients with colorectal cancer, and improving the quality of life of the patients. At this stage, the clinical diagnosis of colorectal cancer is mainly performed by ordinary colonoscopy, but this examination method often encounters missed diagnosis due to the inability to diagnose tiny polyps or flat lesions without distinctive feature, thus delaying the treatment of the patients (Jiang \& Yan, 2017). As an emerging imaging diagnostic technology under endoscope, NBI uses three special narrow-band filters based on traditional RYB imaging, that is, the narrow-band filters filter the broadband light waves in the red, green, and blue spectrums, leaving only the narrowband light waves (Marchand et al., 1997). In addition, NBI does not need staining during the endoscopy, which can better show the micro morphological structure of the mucosal surface of the digestive tract, and can find lesions that cannot be displayed under ordinary endoscopy, thus greatly improving the detection rate of colorectal lesions, and playing an important role in the early screening of colorectal cancer. This research selected 100 patients who underwent colonoscopy in the endoscopy room of our hospital in the past year, and adopted NBI examination. The examination results showed that conducting NBI examination for patients with early colorectal cancer can effectively improve the detection rate of early colorectal precancerous lesions, thus effectively guiding the later treatment and improving the survival rate of patients. Moreover, it has the advantages of simple operation, low examination cost, high safety, and high accuracy, plays a good clinical application value in the early screening of colorectal cancer, reduces the economic burden of the patients, plays a good social value, and is suitable for clinical promotion. The detailed report of this research is as follows.

\section{Data and methods}

\subsection{General data}

200 patients who underwent colonoscopy in the endoscopy room of our hospital in the past year were selected, including 108 male 
patients and 92 female patients, with their ages of 18-80 and an average age of $(55.82 \pm 13.79)$ years. The patients selected for this research should exclude the patients with the family history of colorectal adenoma; the patients with inflammatory bowel disease, such as ulcerative colitis, Crohn's disease, etc.; the patients who are unable to endure the pain of endoscopy, causing the failure of endoscope to reach the ileocecus; the patients whose intestinal tract is not fully prepared and has an impact on the observation under endoscopy. In addition, all examination methods of this research had been approved by the Ethics Committee of our hospital, and all patients who participated in this research and their family members were aware of the contents and plan of this research, voluntarily accepted this research and could offer active cooperation. In this research, all patients were randomized into the test group and control group, and there was no significant difference in the general information between the two groups, such as age, gender, and bowel preparation $(\mathrm{P}>0.05)$.

\section{Method}

All patients received an intramuscular injection of $10 \mathrm{mg}$ of anisodamine before the examination and were fully prepared for the intestinal examination.

\subsection{Control group}

Ordinary endoscopy was performed for the 100 patients in the control group. First, colonoscope was inserted until reaching the ileocecus according to the conventional endoscopy sequence, then the colonoscope was slowly retracted; at the same time, the ordinary colonoscopy mode was used to observe the ileocecus, ascending colon, transverse colon, descending colon, sigmoid colon, rectum, and anus successively. The lesions were sent for pathological examination immediately after being found, and the number of lesions were recorded. Once again, the colonoscope was inserted until reaching the ileocecus according to the conventional endoscopy sequence, when the colonoscope was retracted and after the lesion was found, the gross morphology was recorded and the image definition was evaluated.

\subsection{Test group}

NBI examinations were performed for the 100 patients in the test group. First, the white light mode was used to insert the colonoscope until reaching the ileocecus according to the conventional endoscopy sequence, and then NBI mode was used to slowly retract the colonoscope and observe the ileocecus, ascending colon, transverse colon, descending colon, sigmoid colon, rectum, and anus successively. The lesions were sent for pathological examination immediately after being found, and the number of lesions were recorded. Once again, the colonoscope was inserted until reaching the ileocecus according to the conventional endoscopy sequence, and when the colonoscope was retracted, the morphological structures of the focal pit pattern and superficial capillaries were observed, and the image definition was evaluated.

\subsection{Evaluation indicators}

Evaluation of lesion detection rate: The medical staff participating in this research sorted out the recorded lesion detection data of the two groups of patients, adopted a percentage calculation method to evaluate the lesion detection rates of the two groups of patients, and compare the lesion detection rates of the two groups of patients.

Evaluation of image definition: the two examination methods were evaluated according to the following criteria: 1 : invisible; 2 : blurred but visible; 3: clear; 4: very clear. The image definitions of the examination methods used by the two groups of patients were compared.

Evaluation of lesion detection results: The classification of pit patterns is based on Kudo Shinei classification method (Liu et al., 2003). Type I pit pattern is round, normal pit pattern with a size of $0.07 \pm 0.02 \mathrm{~mm}$; Type II pit pattern is star-shaped or papillary, and belongs to hyperplastic lesion, with a size of $0.09 \pm 0.02 \mathrm{~mm}$; Type III is the pit pattern of adenoid tumor, of which, Type IIIL pattern is tubular or quasi-round with a size of $0.22 \pm 0.09 \mathrm{~mm}$, Type IIIS pattern is relatively uncommon and is small tubular or quasi-round with a size of $0.03 \pm 0.01 \mathrm{~mm}$; Type IV pattern is branched, gyrus-shaped or groove-shaped, and belongs to villous adenoma, with a size of $0.93 \pm 0.32 \mathrm{~mm}$; Type $\mathrm{V}$ pattern is mainly cancer, of which, Type VA pattern is of different sizes and irregular arrangements, and belongs to early cancer, while Type VN pit pattern disappears or has no structure, and is mainly infiltrating cancer. The vessel classification adopts Sano Nei classification method (Tung et al., 2001). Type I capillaries are fuzzy, most of which are normal mucosa or hyperplastic polyps, with a diameter between 6.4-20.9 $\mu \mathrm{M}$; Type II capillaries are clearly visible with slight thickening, most of which are adenomas, with a diameter of $13.1+3.3 \mu \mathrm{M}$; Type III is divided into two subtypes, Type IIIA has visible thicker capillaries with different sizes and irregular branching and shortening, most of which are intramucosal or superficial cancer, while Type IIIB is mostly infiltrating cancer, and the scattered microvessels are almost invisible; the diameter of both types is between $2.2-84.5 \mu \mathrm{M}$. The medical staff participating in this research evaluated the histopathological types of the two groups of patients according to the above methods, and compared the coincidence rates of pathological examinations of the two groups of patients.

\subsection{Statistical analysis}

SPSS17.0 statistical software was used to perform statistical analysis for the data of this research. The enumeration data was described by $\mathrm{n}(\%)$, the comparison among groups was performed by $\mathrm{x}^{2}$ test; the measurement data was described by $(\overline{\mathrm{x}} \pm \mathrm{s})$, and the comparison among groups was performed by t test. The test criterion is $\alpha=0.05$, when $\mathrm{P}<0.05$, the comparative difference between the two groups is statistically significant.

\section{Results}

\subsection{Comparison of the lesion detection rate between two groups of patients}

Table 1 lists the analysis results of lesion detection rates of the two research groups, the lesion detection rate of the test group is significantly higher than that of the control group $(77.0 \%$ vs 
$28.0 \%$ respectively, $\mathrm{P}<0.05$ ), and the comparative difference between two groups is statistically significant.

\subsection{Comparison of image definition between the two groups of patients}

Table 2 lists the analysis results of the image definition scores of the two research groups, the image definition score of the test group is significantly higher than that of the control group $(3.46 \pm 0.50 \mathrm{vs}$ $2.41 \pm 1.16$ respectively, $\mathrm{P}<0.001$ ), and the comparative difference between two groups is statistically significant.

\subsection{Comparison of pathological examination results between the two groups of patients}

Table 3 lists the analysis results of the pathological examination results of the two research groups, the lesion detection rates of hyperplastic lesion ( $13.0 \%$ vs 5.0\%, $\mathrm{P}<0.05)$, tubular adenoma ( $19.0 \%$ vs $9.0 \%, \mathrm{P}<0.05)$, villous adenoma ( $12.0 \%$ vs $4.0 \%, \mathrm{P}<0.05)$, tubular villous adenoma ( $16.0 \%$ vs $7.0 \%, \mathrm{P}<0.05)$, early cancer $(9.0 \%$ vs $2.0 \%, \mathrm{P}<0.05)$ and infiltrating cancer $(8.0 \%$ vs $1.0 \%, \mathrm{P}<0.05)$ of the test group are significantly higher than those of the control group, and the comparative difference between two groups is statistically significant. Therefore, compared with the control group, the test group has a higher coincidence rate of lesion detection.

\section{Discussion}

Colorectal cancer is a relatively common malignant tumor disease in clinical practice. Nowadays, with the continuous improvement of people's living standards and the increasingly

Table 1. Comparison of the number of lesions detected in the two groups of patients, $\mathrm{n}(\%)$.

\begin{tabular}{cccc}
\hline Group & $\begin{array}{c}\text { Number of } \\
\text { cases }(\mathrm{n})\end{array}$ & Detected & Not detected \\
\hline Test group & 100 & $77(77.0)$ & $23(23.0)$ \\
Control group & 100 & $28(28.0)$ & $72(72.0)$ \\
$\mathrm{x}^{2}$ & & 48.140 & \\
$\mathrm{P}$ & & 0.000 & \\
\hline
\end{tabular}

Table 2. Comparison of image definition scores of the two examination methods (points, $\bar{x} \pm s$ ).

\begin{tabular}{ccc}
\hline Group & Number of cases & Image definition score \\
\hline Test group & 100 & $3.46 \pm 0.50$ \\
Control group & 100 & $2.41 \pm 1.16$ \\
t value & & 8.285 \\
P value & & $<0.001$ \\
\hline
\end{tabular}

westernized diet culture, the morbidity of colorectal cancer is increasing, posing a serious threat to human health. At this stage, the clinical pathogenesis of colorectal cancer has not yet been clarified, but in-depth researches have been conducted on the pathogenetic factors, and it is clinically believed that the pathogenesis of colorectal cancer is closely related to living habits, environment, diet, and genetic factors (Han et al., 2016). Related research has shown that screening can realize the early detection, early diagnosis and early treatment of colorectal cancer, thus increasing the effective rate of treatment and improving the prognosis (Liu \& Zao, 2018). Therefore, early screening plays an important role in the timely detection and effective treatment of colorectal cancer and precancerous lesions (Xiong et al., 2012). For a long time, the method that is clinically used for the longest time and of the highest reliability is ordinary endoscopy combined with mucosal biopsy, but this method has the disadvantage of high rate of missed diagnosis, which prevents the doctors from detecting the condition early, and then delays treatment and reduces survival rate (Ren et al., 2016). As an emerging endoscopic imaging technology, NBI retains all the functions of ordinary endoscopy, and uses the special narrow-spectrum filter in the endoscopic system to more clearly show the morphology of microvessels on the mucosal surface and the morphology of the micro glandular tubes, and improve the visibility of the morphology of the glandular tubes and vascular structure of large intestine mucosa (Zhou et al., 2011). Moreover, it is of simple operation and does not need staining during the examination, which can avoid misjudgment of lesions due to uneven distribution of stains and limited observation field, and can avoid the side effects of stains and reduce unnecessary harm to the patients (Emura et al., 2008), thus realizing higher detection rate and accuracy.

In order to explore the clinical application value of narrow band imaging technology in the early screening of colorectal cancer, this research compared the lesion detection rate, image definition and pathological detection coincidence rate of the control group using ordinary endoscopy and the test group using NBI examination. The results showed that 77 lesions were detected in the test group (77.0\%), and 28 lesions were detected in the control group (28.0\%), the lesion detection rate of the test group was significantly higher than that of the control group, and the difference between the two groups was significant $(\mathrm{P}<0.05)$; the image definition score of the test group was $3.46 \pm 0.50$ points, the image definition score of the control group was $2.41 \pm 1.16$ points, so the image definition of the test group was significantly higher than that of the control group, and the difference between the two groups was significant $(\mathrm{P}<0.001) ; 13$ cases of hyperplastic lesion $(13.0 \%), 19$ cases of tubular adenoma (19.0\%), 12 cases of villous adenoma (12.0\%), 16 cases of tubular villous adenoma ( $16.0 \%), 9$ cases of early cancer (9.0\%) and 8 cases of infiltrating cancer (8.0\%) were detected in

Table 3. Comparison of lesion detection results between the two groups of patients, $n$ (\%).

\begin{tabular}{cccccccc}
\hline Group & $\begin{array}{c}\text { Number of cases } \\
(\mathrm{n})\end{array}$ & $\begin{array}{c}\text { Hyperplastic } \\
\text { lesion }\end{array}$ & $\begin{array}{c}\text { Tubular } \\
\text { adenoma }\end{array}$ & Villous adenoma & $\begin{array}{c}\text { Tubular villous } \\
\text { adenoma }\end{array}$ & $\begin{array}{c}\text { Early cancer } \\
\text { Infiltrating } \\
\text { cancer }\end{array}$ \\
\hline Test group & 100 & $13(13.0)$ & $19(19.0)$ & $12(12.0)$ & $16(16.0)$ & $9(9.0)$ & $8(8.0)$ \\
Control group & 100 & $5(5.0)$ & $9(9.0)$ & $4(4.0)$ & $7(6.0)$ & $2(2.0)$ & $1(1.0)$ \\
$\mathrm{x}^{2}$ & & 3.889 & 4.121 & 4.330 & 3.955 & 4.701 & 5.688 \\
$\mathrm{P}$ & & 0.049 & 0.042 & 0.037 & 0.047 & 0.030 & 0.017 \\
\hline
\end{tabular}


the test group, while 5 cases of hyperplastic lesions (5.0\%), 9 cases of tubular adenoma (9.0\%), 4 cases of villous adenoma (4.0\%), 7 cases of tubular villous adenoma (7.0\%), 2 cases of early cancer (2.0\%) and 1 case of infiltrating cancer (1.0\%) were detected in the control group; the coincidence rate of pathological detection in the test group was significantly higher than that of the control group, and the difference between the two groups was significant $(\mathrm{P}<0.05)$. Therefore, practice has been proved that compared with ordinary endoscopy, the NBI examination has higher resolution and higher image definition, can effectively display the mucosal vascular network, clearly show the pit pattern and vascular structure of the large intestine mucosa, which can help find the smaller mucosal tissue lesions, thus greatly improving the lesion detection rate. Moreover, the classification and diagnosis of the pit pattern of large intestinal mucosa are of great significance in determining whether it is early cancer. The research results prove that the NBI examination can better observe the pit pattern of the mucosal glandular tube and the capillary morphology, and can detect tumorous lesions and early cancer as soon as possible, effectively improve the diagnosis rate of early colorectal cancer and precancerous lesions, and play an important role in the effective treatment of colorectal cancer and precancerous lesions.

Food and nutrition are closely related to the incidence of human CRC. Appropriate diet could reduce cancer risk and inhibit tumor growth. For example, synbiotic sheep milk ice cream containing prebiotic and probiotic ingredients could reduce chemically induced colon carcinogenesis (Balthazar et al., 2021). A meta-analysis based on clinical research also suggests that the consumption of fermented dairy products can significantly reduce the risk of precursors of CRC (Guo et al., 2021). Cheddar cheese peptide extracts suppresses lung cancer cells by inducing apoptosis and also suppresses colon adenocarcinoma cells by dose-dependent inducing apoptosis effect (Rafiq et al., 2018, 2020). Therefore, it is of great clinical significance in decreasing incidence of CRC in high CRC risk areas by changing of dietary habits (Papadimitriou et al., 2021).

In summary, the NBI examination of patients can clearly show the pit pattern and the capillary morphology of the early colorectal cancer and its precancerous lesions, thus effectively improving the diagnosis rate of early colorectal cancer and precancerous lesions, and guiding the later treatment, has played a good clinical application value in the early screening of colorectal cancer, so it is suitable for clinical promotion.

\section{References}

Balthazar, C. F., Moura, N. A., Romualdo, G. R., Rocha, R. S., Pimentel, T. C., Esmerino, E. A., Freitas, M. Q., Santillo, A., Silva, M. C., Barbisan, L. F., Cruz, A. G., \& Albenzio, M. (2021). Synbiotic sheep milk ice cream reduces chemically induced mouse colon carcinogenesis. Journal of Dairy Science, 104(7), 7406-7414. http:// dx.doi.org/10.3168/jds.2020-19979. PMid:33934866.

Emura, F., Saito, Y., \& Ikematsu, H. (2008). Narrow-band imaging optical chromocolonoscopy: advantages and limitations. World Journal of Gastroenterology, 14(31), 4867-4872. http://dx.doi.org/10.3748/ wjg.14.4867. PMid:18756593.

Guo, L. L., Li, Y. T., Yao, J., Wang, L. S., Chen, W. W., He, K. Y., Xiao, L., \& Tang, S. H. (2021). Dairy consumption and risk of conventional and serrated precursors of colorectal cancer: a systematic review and meta-analysis of observational studies. Journal of Oncology, 2021(1), 9948814. PMid:34122548.

Han, Y., Juan, M., \& Yi, Z. (2016). Analysis of the clinical value of endoscopic narrow band imaging technology in the diagnosis of early colorectal cancer. Modern Digestion \& Intervention, 21(4), 583.

Jiang, C., \& Yan, J. (2017). The value of narrow band imaging (NBI) targeted biopsy in the screening of early colorectal cancer and precancerous lesions. Jilin Medical Journal, 38(9), 1691-1692.

Liu, H. H., Kudo, S. E., \& Juch, J. P. (2003). Pit pattern analysis by magnifying chromoendoscopy for the diagnosis of colorectal polyps. Journal of the Formosan Medical Association, 102(3), 178-182. PMid:12783135.

Liu, Z., \& Zhao, J. (2018). Application research on magnifying endoscopy with narrow band imaging technology in the diagnosis of digestive tract early cancer and precancerous lesions in the elderly. Clinical Journal of Medical Officer, 46(8), 983-984.

Marchand, L. L., Wilkens, L. R., Hankin, J. H., Kolonel, L. N., \& Lyu, L. C. (1997). A case-control study of diet and colorectal cancer in a multiethnic population in Hawaii (United States): lipids and foods of animal origin. Cancer Causes \& Control, 8(4), 637-648. http:// dx.doi.org/10.1023/A:1018406716115. PMid:9242481.

Papadimitriou N, Bouras E, Van Den Brandt PA, Muller DC, Papadopoulou A, Heath AK, Critselis E, Gunter MJ, Vineis P, Ferrari P, Weiderpass E, Boeing H, Bastide N, Merritt MA, Lopez DS, Bergmann MM, Perez-Cornago A, Schulze M, Skeie G, Srour B, Eriksen AK, Boden S, Johansson I, Nøst TH, Lukic M, Ricceri F, Ericson U, Huerta JM, Dahm CC, Agnoli C, Amiano PE, Tjønneland A, Gurrea AB, Bueno-de-Mesquita B, Ardanaz E, Berntsson J, Sánchez MJ, Tumino R, Panico S, Katzke V, Jakszyn P, Masala G, Derksen JWG, Quirós JR, Severi G, Cross AJ, Riboli E, Tzoulaki I, Tsilidis KK. (2021). A prospective diet-wide association study for risk of colorectal cancer in EPIC. Clinical Gastroenterology Hepatology, (21), 462-466.

Rafiq, S., Gulzar, N., Huma, N., Hussain, I., \& Murtaza, M. S. (2020). Evaluation of anti-proliferative activity of Cheddar cheeses using colon adenocarcinoma (HCT-116) cell line. International Journal of Dairy Technology, 73(1), 255-260. http://dx.doi.org/10.1111/1471-0307.12665.

Rafiq, S., Huma, N., Gulzar, N., Murtaza, M. A., \& Hussain, I. (2018). Effect of Cheddar cheese peptide extracts on growth inhibition, cell cycle arrest and apoptosis induction in human lung cancer (H-1299) cell line. International Journal of Dairy Technology, 71(4), 975-980. http://dx.doi.org/10.1111/1471-0307.12533.

Ren, D., Ping, X., Kai, X., \& Jing, W. (2016). Application value of magnifying endoscopic narrow band imaging combined with high-frequency intracavitary ultrasound microprobe in minimally invasive diagnosis and treatment of wide-fundus/laterally developed lesions of large intestine. Chinese Journal of Clinical Gastroenterology, 28(03), 137.

$\mathrm{Tu}, \mathrm{J}$. (2019). Application and clinical value of colonoscopy biopsy in the diagnosis of colorectal cancer. Chinese Journal of Integrated Traditional Chinese and Western Medicine on Digestion, 27(3), 66-69.

Tung, S. Y., Wu, C. S., \& Su, M. Y. (2001). Magnifying colonoscopy in differentiating neoplastic from nonneoplastic colorectal lesions. The American Journal of Gastroenterology, 96(9), 2628-2632. http:// dx.doi.org/10.1111/j.1572-0241.2001.04120.x. PMid:11569686.

Xiong, J., Song, F., Kuang, G., Yan, D., Xiao, S., \& Fu, X. (2012). The clinical application of narrow band imaging technology in the diagnosis and treatment of early colorectal cancer and precancerous lesions. Contemporary Medicine, 18(33), 154-155.

Xu, D., Li, S., Lv, S., Gao, T., Zhang, S., Xu, J., \& Chen, N. (2018). The clinical value of narrow band imaging technology combined with chromoendoscopy in the diagnosis of early cancer and precancerous lesions of the digestive tract. Chinese Journal of the Frontiers of Medical Sciences, 10(10), 94-97. 
Yan, H., Lei, G. Lv, W., Liu, M., Zhao, X., Yao, S., Yang, Y., Shan, J., \& Liu, Z. (2016). Significance of narrow band imaging combined with magnifying endoscopy in the qualitative diagnosis of gastric mucosal lesions. Chinese Journal of Digestive Endoscopy, 33(9), 609.
Zhou, Q., Yang, J., Fei, B., Xu, Q., Wu, W., Ruan, H. H. (2011). Narrowband imaging endoscopy with and without magnification in diagnosis of colorectal neoplasia. China Academic Journal Electronic Publishing House, 17(5), 666-670. 